\title{
Clinical Application of the Threshold Equalizing Noise Test in Patients with Hearing Loss of Various Etiologies: A Preliminary Study
}

\author{
Ho Yun Lee, Yu Mi Seo, Kyung Ae Kim, Yeon Shin Kang, and Chin Saeng Cho \\ Department of Otorhinolaryngology, Eulji University Medical Center, Eulji University, Daejeon, Korea
}

Received November 4, 2014

Revised December 17, 2014

Accepted January 5, 2015

\section{Address for correspondence Ho Yun Lee, MD, PhD \\ Department of Otorhinolaryngology, Eulji University Medical Center, Eulji University, 95 Dunsanseo-ro, Seo-gu, Daejeon 302-799, Korea \\ Tel $+82-42-611-3133$ \\ Fax +82-42-611-3136 \\ E-mail hoyun1004@gmail.com}

Background and Objectives: We aimed to make a preliminary assessment of the prevalence of cochlear dead regions (DRs) and the factors affecting the results of the threshold-equalizing noise (TEN) test in patients with hearing loss of various etiologies. Subjects and Methods: Between May and July 2014, 109 patients (191 ears) with hearing loss who visited our outpatient clinic were prospectively enrolled. Pure tone audiometry and TEN $(\mathrm{HL})$ test were performed for all the patients. DR at each frequency was indicated by masked thresholds of $\geq 10$ $\mathrm{dB}$ above the TEN level and $\geq 10 \mathrm{~dB}$ above the absolute threshold. Results: DR was present in $15.7 \%$ ( $n=30$ ) of the 191 ears. According to disease entity, $16.6 \%$ of patients with sensorineural hearing loss had a DR. However, DR was absent in patients with chronic otitis media. According to audiometric configurations, DR was most common in moderately severe, flat hearing loss. Significantly worse hearing thresholds for both mean hearing level and hearing threshold at each frequency were found in the presence of DR $(p<0.001)$. Logistic regression analysis showed that only the mean hearing level (odds ratio: 1.053, 95\% confidence interval: 1.021-1.085) affected the presence of DR. Conclusions: Although performance of the TEN test is limited by frequencies and hearing levels, it provides additional information regarding DRs and may therefore have the potential to be used as a prognostic tool for diverse diseases causing hearing loss.

J Audiol Otol 2015;19(1):20-25

KEY WORDS: Threshold equalizing noise test · Hearing loss · Cochlear dead regions.

\section{Introduction}

Cochlear dead regions (DRs) are defined as areas of inner hair cells and/or related neurons, which may not function normally in hearing loss at a given frequency [1]. Basilar membrane vibration may not be detected by auditory neurons within frequencies including DRs, and off-place listening can occur around the DR; therefore, the actual severity of hearing loss may be worse than the estimated thresholds by pure tone audiometry.

The threshold-equalizing noise (TEN) test is designed to detect conveniently the presence of DRs in clinical settings.

This is an Open Access article distributed under the terms of the Creative Commons Attribution Non-Commercial License (http://creativecommons. org/licenses/by-nc/3.0/) which permits unrestricted non-commercial use, distribution, and reproduction in any medium, provided the original work is properly cited.
The earlier version of the TEN test was calibrated in $\mathrm{dB}$ sound pressure level (SPL) and referred to as the TEN (SPL) test [1]. The second version of the TEN test was designed to provide approximately the same masked pure tone thresholds in $\mathrm{dB} \mathrm{HL}$ for wide frequencies $(500-4000 \mathrm{~Hz})$ in normal hearers and is referred to as the TEN (HL) test [2,3]. In patients with DRs, the TEN-masked threshold at the specific frequency with $\mathrm{DR}$ is expected to be higher than that in normal hearers [4]. A 10-dB increase in the masked threshold above the TEN level and absolute hearing threshold is indicative of DR [2-4].

Recent studies have reported variations in the results of the TEN test according to hearing aids fitting as well as specific inclusion criteria such as audiometric patterns, confined to sensorineural hearing loss, and specific thresholds range [1-6]. However, all patients who visit the clinic with a complaint of hearing loss do not show always sensorineural hearing loss confined to a specific range of pure tone thresholds or bilat- 
eral hearing loss; in fact, some do not even expect to be prescribed hearing aids for unconditional use.

The aim of this study was to make a preliminary assessment of the prevalence of DRs and the factors influencing the results of the TEN (HL) test in patients with hearing loss of various etiologies, not confined to sensorineural hearing loss.

\section{Subjects and Methods}

Between May and July 2014, we prospectively enrolled 109 patients (191 ears) with hearing loss who visited the outpatient clinic at Eulji University Hospital and agreed to participate in this study. The Institutional Review Board of Eulji University Hospital approved this study.

The following patient information was recorded: age, gender, presence of diabetes or hypertension, presence of tinnitus, and/or dizziness. One of three audiologists with more than five years' experience performed pure tone audiometry and the TEN (HL) test using the MADSEN astera (GN Otometrics, Copenhagen, Denmark) with TDH-39 headphones in the sound booth. Pure tone air and bone thresholds were obtained at $0.25,0.5,1.0,2.0,4.0$, and $8.0 \mathrm{kHz}$, and the TEN (HL) test was performed at $0.5 \mathrm{~Hz}, 1 \mathrm{kHz}, 2 \mathrm{kHz}$, and $4 \mathrm{kHz}$. The mean hearing level was calculated using the arithmetic mean of the pure tone thresholds at $0.5,1,2$, and $4 \mathrm{kHz}$.

For frequencies at which the absolute hearing threshold was $\leq 60 \mathrm{~dB}$ HL, the TEN level was set to $70 \mathrm{~dB}$ HL [3]. If the hearing loss exceeded $70 \mathrm{~dB}$ HL, the TEN level was set to $10 \mathrm{~dB}$ above the audiometric threshold at each frequency, up to a maximum of $86 \mathrm{~dB}$ HL. If the patient could not tolerate the loudness of the TEN, or if the maximal TEN level was reached, the TEN level was set equal to the audiometric threshold [2,3]. The signal level was increased in 2-dB step size to determine the thresholds [2-6].

At each frequency, DR was detected according to criteria suggested by Moore, et al. [2]. DR was assumed to be present if the masked threshold was $\geq 10 \mathrm{~dB}$ above the TEN level and $\geq 10 \mathrm{~dB}$ above the absolute threshold. DR was assumed to be absent if the masked threshold was $<10 \mathrm{~dB}$ above the TEN level and $\geq 10 \mathrm{~dB}$ above the absolute threshold. DR was considered inconclusive if the masked threshold was $<10 \mathrm{~dB}$ above the absolute threshold and the signal level could not be increased any further [6].

The audiometric configurations were classified as low-frequency hearing loss, high-frequency hearing loss, flat hearing loss, and total hearing loss [7]. Low-frequency hearing loss was defined as normal hearing that was maintained at 4.0 $\mathrm{kHz}$ or $8.0 \mathrm{kHz}$, a low tone tilt with a difference of $25 \mathrm{~dB}$ between $0.5 \mathrm{kHz}$ and $4 \mathrm{kHz}$, or the presence of a high tone loss in the other ear. High-frequency hearing loss was defined as normal hearing that was maintained at 0.25 or $0.5 \mathrm{kHz}$, or a high tone tilt to the audiogram with a difference of $25 \mathrm{~dB}$ between 0.5 and $4 \mathrm{kHz}$. Flat hearing loss was determined when the average hearing loss at $0.5-2 \mathrm{kHz}$ did not exceed $90 \mathrm{~dB}$ and the audiometric pattern did not fall into low tone or high tone hearing loss categories. Total hearing loss was determined when the average hearing loss exceeded $90 \mathrm{~dB}$ [7]. The mean hearing levels were classified into six groups following the International Organization for Standardization (ISO 1964): normal, $-25 \mathrm{~dB}$; mild hearing loss, 26-40 dB; moderate hearing loss, 41-55 dB; moderately severe hearing loss, 56-70 dB, severe hearing loss, 71-90 dB; and profound hearing loss, $\geq 91 \mathrm{~dB}$.

For statistical analyses, binary logistic regression analysis was conducted to identify the factors influencing the TEN (HL) test results among the factors identified by univariate analysis. All statistical analyses were performed using SPSS software (ver. 18.0, SPSS Inc., Chicago, IL, USA) and R Commander Plug-in for the EZR Package (RcmdrPlugin.EZR), and a $p$-value of $<0.05$ was considered significant [8].

\section{Results}

The baseline characteristics of the 109 patients (191 ears) included in this study are listed in Table 1 . A total of 24 pa-

Table 1. Patients' characteristics

\begin{tabular}{lc}
\hline \multicolumn{1}{c}{ Factors } & Values \\
\hline Age (years old) & $56.29 \pm 14.725$ (range 15-84) \\
Sex (male/female) & $58 / 51$ \\
Diabetes & $6(5.5)$ \\
Hypertension & $28(25.7)$ \\
Accompanying tinnitus & $55(50.5)$ \\
Accompanying dizziness & $18(16.5)$ \\
Mean hearing level (dB) & $42.58 \pm 25.23$ \\
Audiometric configurations & \\
Low-frequency hearing loss & $7(3.7)$ \\
High-frequency hearing loss & $100(52.4)$ \\
Flat hearing loss & $75(39.3)$ \\
Total hearing loss & $9(4.7)$ \\
Initial diagnosis & \\
Acoustic trauma & $1(0.9)$ \\
Chronic otitis media & $8(7.3)$ \\
Herpes zoster oticus & $2(1.8)$ \\
Meniere's disease & $7(6.4)$ \\
Sensorineural hearing loss & $77(70.6)$ \\
Sudden hearing loss & $14(12.8)$ \\
\hline Data were shown as the mean &
\end{tabular}

Data were shown as the mean \pm SD for continuous variables and number (\%) for categorical variables. SD: standard deviation 
tients $(22.1 \%)$ had a DR at one or more frequencies. Of the 191 ears, DR was detected in 30 ears (15.7\%). Moreover, 28 patients (34 ears) showed an inconclusive result because of limit in the highest signal level at each frequency. Of these, 7 patients ( 7 ears) had both DR and inconclusive DR according to frequency.

According to the mean hearing level (Table 2), DR was the most common in patients with moderately severe hearing loss (50.0\%), followed by those with severe hearing loss $(33.3 \%)$, and moderate hearing loss (13.6\%). In contrast, inconclusive DR was the most common in patients with profound hearing loss (100.0\%), followed by those with severe hearing loss (76.2\%), and moderately severe hearing loss (17.9\%).

The presence of DR according to the hearing level showed a significant difference $(p<0.001)$ (Table 2$)$. In addition, the hearing threshold at each frequency with a DR was significantly higher than that at frequencies without a DR $(p<0.05)$ (Fig. 1).

With regard to audiometric configurations (Table 3), flat hearing loss was the most common in patients with both DR and inconclusive DR. High-frequency hearing loss was the second most common in patients with DR; however, total hearing loss was the second most frequent in patients with inconclusive DR

With regard to frequencies (Table 4), DR was the most common at $4000 \mathrm{~Hz}(n=20)$, followed by $1000 \mathrm{~Hz}(n=13), 2000$
$\mathrm{Hz}(\mathrm{n}=11)$, and $500 \mathrm{~Hz}(\mathrm{n}=7)$. The number of frequencies that showed a DR was one $(n=19)$, two $(n=4)$, three $(n=4)$, and all four frequencies $(n=3)$. The overall detection rate of DR below $60 \mathrm{~dB}$ was $0.9 \%$.

With regard to the disease diagnosed in the patient (Table 5), $16.6 \%$ of patients with sensorineural hearing loss had a DR. Similarly, DR was present in $14.3 \%$ of patients diagnosed with

Table 3. Results of the threshold equalizing noise test according to audiometric configuration

\begin{tabular}{lcc}
\hline Audiometric configuration & Positive DR* & $\begin{array}{c}\text { Inconclusive } \\
\text { DR* }\end{array}$ \\
\hline Low-frequency hearing loss & $0(0.0)$ & $0(0.0)$ \\
High-frequency hearing loss & $8(26.7)$ & $5(14.7)$ \\
Mild hearing loss & $4 / 8$ & $2 / 5$ \\
Moderate hearing loss & $1 / 8$ & $2 / 5$ \\
Moderately severe hearing loss & $2 / 8$ & $0 / 5$ \\
Severe hearing loss & $1 / 8$ & $1 / 5$ \\
Flat hearing loss & $21(70.0)$ & $20(58.8)$ \\
Mild hearing loss & $1 / 21$ & $0 / 20$ \\
Moderate hearing loss & $2 / 21$ & $0 / 20$ \\
Moderately severe hearing loss & $12 / 21$ & $5 / 20$ \\
Severe hearing loss & $6 / 21$ & $15 / 20$ \\
\hline Total hearing loss & $1(3.3)$ & $9(26.5)$ \\
\hline
\end{tabular}

Data were shown as number (\%) for categorical variables and number of affected ears/total numbers of ears for adjusting hearing levels. $* p<0.05$. DR: cochlear dead region

Table 2. Results of the threshold equalizing noise test according to degree of hearing loss

\begin{tabular}{lccc}
\hline \multicolumn{1}{c}{ Degree of hearing loss* } & Number of ears & Positive DR & Inconclusive DR \\
\hline Normal hearing $(\leq 25 \mathrm{~dB})$ & 62 & $0(0.0)$ & $0(0.0)$ \\
Mild hearing loss $(26-40 \mathrm{~dB})$ & 49 & $5(10.2)$ & $2(4.1)$ \\
Moderate hearing loss $(41-55 \mathrm{~dB})$ & 22 & $3(13.6)$ & $2(9.1)$ \\
Moderately severe hearing loss $(56-70 \mathrm{~dB})$ & 28 & $14(50.0)$ & $5(17.9)$ \\
Severe hearing loss $(71-90 \mathrm{~dB})$ & 21 & $7(33.3)$ & $16(76.2)$ \\
Profound hearing loss $(\geq 91 \mathrm{~dB})$ & 9 & $1(11.1)$ & $9(100.0)$
\end{tabular}

Data were shown as number (\%) for categorical variables. *degree of hearing loss was classified following the International Organization for Standardization (ISO 1964), ${ }^{\dagger} p<0.001$. DR: cochlear dead regions

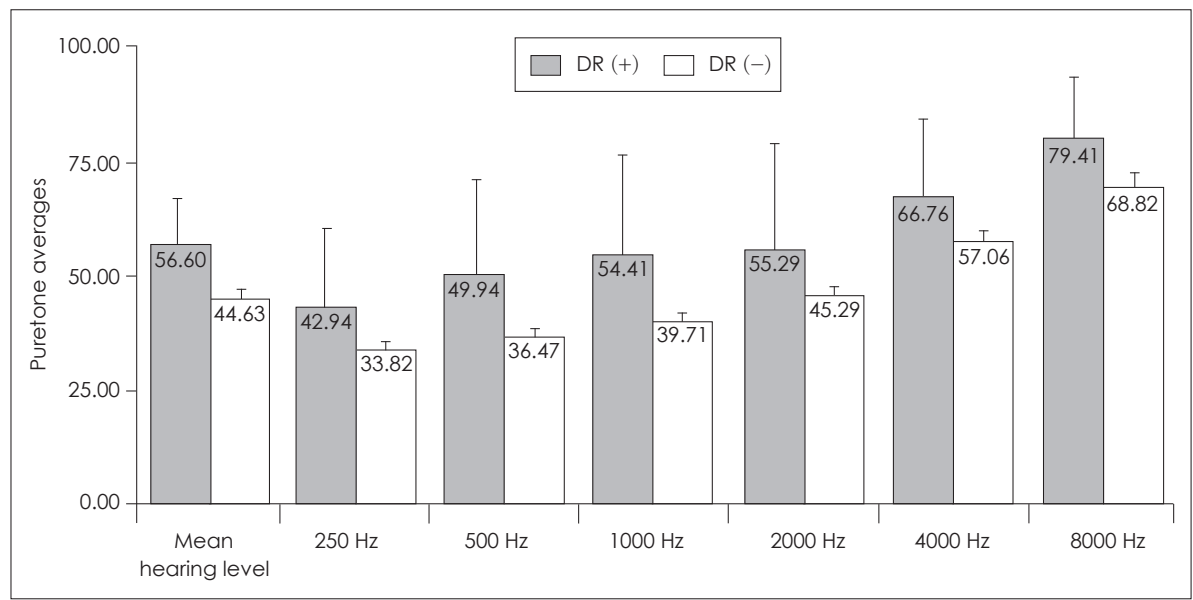

Fig. 1. Hearing threshold at each frequency according to the presence of cochlear dead regions (DRs). 
sudden hearing loss. In contrast, DR was absent in cases of chronic otitis media and Meniere's disease.

Finally, binary logistic regression analysis showed that the mean hearing level was the sole factor that influenced the presence of DR (Table 6).

\section{Discussion}

In this study, we assessed the prevalence of DRs and the factors influencing the results of the TEN (HL) test in patients with hearing loss of various etiologies. The strength of this study is that, for the first time in Korea, the TEN (HL) test was performed for patients with not only sensorineural hearing loss for hearing aid fitting but also hearing loss of various eti-

Table 4. Distribution of pure tone thresholds and cochlear dead regions

\begin{tabular}{ccccc}
\hline $\mathrm{dB}$ & $500 \mathrm{~Hz}$ & $1000 \mathrm{~Hz}$ & $2000 \mathrm{~Hz}$ & $4000 \mathrm{~Hz}$ \\
\hline 0 & 5 & 3 & 2 & 5 \\
5 & 12 & 14 & 9 & 2 \\
10 & 23 & 11 & 13 & 7 \\
15 & 25 & 25 & 13 & 11 \\
20 & 17 & 18 & 15 & 1 \\
25 & 16 & 11 & 14 & 14 \\
30 & 13 & 15 & 18 & $9(1)$ \\
35 & 11 & 9 & 10 & 11 \\
40 & 7 & 12 & 11 & 15 \\
45 & 10 & 10 & 11 & $12(1)$ \\
50 & 2 & $5(1)$ & 8 & 12 \\
55 & $7(1)$ & 5 & $10(1)$ & $10(1)$ \\
60 & 3 & $5(1)$ & $7(3)$ & $10(1)$ \\
65 & $7(1)$ & $6(1)$ & $5(1)$ & $8(1)$ \\
70 & $1(1)$ & $6(5)$ & 7 & $10(4)$ \\
75 & $2(1)$ & $3(2)$ & $10(3)$ & $7(5)$ \\
80 & $7(3)$ & $3(2)$ & $3(3)$ & $5(4)$ \\
85 & 2 & $5(1)$ & 2 & $5(2)$ \\
\hline Total & $170(7)$ & $166(13)$ & $168(11)$ & $154(20)$ \\
\hline
\end{tabular}

The first entry in each cell indicates the number of ears having pure tone thresholds for each frequency. Numbers in parenthesis indicates the number of ears meeting criteria of cochlear dead regions. No number in parenthesis indicates the cases that dead region was absent.

Table 5. Difference in the presence of cochlear dead regions according to disease entity

\begin{tabular}{lcc}
\hline \multicolumn{1}{c}{ Disease } & $\begin{array}{c}\text { Mean hearing } \\
\text { level }(\mathrm{dB})\end{array}$ & $\begin{array}{c}\text { Number of } \\
\text { DRs/total ears }\end{array}$ \\
\hline Acoustic trauma & 63.75 & $1 / 1$ \\
Chronic otitis media & $63.38 \pm 15.18$ & $0 / 10$ \\
Herpes zoster oticus & $53.13 \pm 22.10$ & $1 / 2$ \\
Meniere's disease & $26.07 \pm 16.48$ & $0 / 7$ \\
Sensorineural hearing loss & $40.69 \pm 24.88$ & $26 / 157$ \\
Sudden hearing loss & $54.20 \pm 28.91$ & $2 / 14$ \\
\hline
\end{tabular}

DRs: cochlear dead regions ologies. We found that $22.1 \%$ patients $(15.7 \%$ of the total 191 ears) with hearing loss who visited the outpatient clinic during the study period had a DR. Compared to previous studies reporting the prevalence of DR $(31-57.4 \%$ based on patients, $23-46 \%$ based on ears), the prevalence of DR appears low in our study [4-6]. We argue that the reason for this low prevalence is that we performed a TEN test in all patients with hearing loss, regardless of the severity.

Interestingly, the prevalence of DR varied according to the final diagnosis of the patient (Table 5). Although patients with chronic otitis media showed the second highest pure tone thresholds, the highest being associated with acoustic trauma, DR was not detected in any of these patients. To our knowledge, the TEN test had not been previously performed in any of these patients. The region of the inner ear with hair cells responsible for high frequency sounds may be vulnerable to chronic otitis media [9]. Based on the results of this preliminary study, we assumed that the possibility of inner ear damage in chronic otitis media may not be too high or may be limited to specific regions. However, this assumption should be verified by further studies.

In this study, we found that DR was the most prevalent in the moderately severe hearing loss group (50.0\%) (Table 2). On the other hand, inconclusive DR was more commonly found, rather than DR, in patients with profound hearing loss (100.0\%). Of the 191 ears, 17.8\% (34 ears) showed inconclusive DR and those were quite common. Thus, we couldn't ignore these inconclusive results and we analysed these independently, not included in assessing DR. Although hearing loss of more than $90 \mathrm{~dB}$ is associated with the presence of DR [10], additional psychophysical tuning curves are not feasible to confirm DR in clinical settings. Thus, inconclusive DR is generally determined in patients with severe-to-profound hear-

Table 6. Results of univariate and logistic regression analyses for detection of cochlear dead regions

\begin{tabular}{lcccc}
\hline Variable & $\begin{array}{c}\text { P value for } \\
\text { univariate } \\
\text { analysis }\end{array}$ & $\begin{array}{c}\text { p value for } \\
\text { logistic } \\
\text { regression }\end{array}$ & $\begin{array}{c}\text { Odds } \\
\text { ratio }\end{array}$ & $\begin{array}{c}95 \% \\
\text { confidence } \\
\text { interval }\end{array}$ \\
\hline Age & 0.433 & - & - & - \\
Sex & 0.215 & - & - & - \\
Diabetes & 0.065 & 0.090 & 3.533 & $0.820-15.217$ \\
Hypertension & 0.779 & - & - & - \\
Tinnitus & 0.412 & - & - & - \\
Dizziness & 0.740 & - & - & - \\
Affected side & 0.778 & - & - & - \\
Mean hearing & $<0.001$ & 0.001 & 1.053 & $1.021-1.085$ \\
level & & & & \\
Audiogram & 0.004 & 0.158 & 2.278 & $0.726-7.143$ \\
pattern & \\
The mean hearing level was calculated using the arithmetic \\
mean of the pure tone thresholds at 0.5, 1, 2, and $4 \mathrm{kHz}$
\end{tabular}


ing loss despite the high possibility of DR-even the determination of inconclusive DR is only possible at narrow frequencies between $500 \mathrm{~Hz}$ and $4000 \mathrm{~Hz}$. This may be considered a limitation of the TEN (HL) test, which can be overcome in future studies.

Patients with flat hearing loss, especially with moderately severe hearing loss adjusted according to the hearing level, showed the highest prevalence of DR, which is in contrast to the results of a previous study that reported a close relationship between steep-sloping hearing loss and the presence of DR [11]. This difference may be attributed to the ambiguity of the audiometric criteria used [7] and worse hearing thresholds in patients with flat type hearing loss. Likewise, Pepler, et al. [5] reported that the slope may only increase the prevalence of DR according to increasing hearing impairment. Accordingly, in our study, logistic regression analysis revealed that the audiometric pattern was not associated with the presence of DR.

DR was the most frequently detected at $4000 \mathrm{~Hz}$, which is consistent with the findings of a previous study [5]. This may suggest that the presence of DR may vary according to the frequency. In addition, an isolated DR, limited to one frequency, was the most common according to the number of affected frequencies, which is similar to the findings of Hornsby and Dundas [12], although the clinical significance of this finding remains unknown. For hearing aids fitting, application of TEN (HL) test at $2 \mathrm{kHz}$ or below is recommended on the basis that amplification should be provided for frequencies up to 1.7 times the edge frequency of DR and the TEN test results at $3 \mathrm{kHz}$ or above may not influence the audibility $[5,6]$. On the contrary, few studies report the significance of TEN test results in the management of hearing loss, unless hearing aid fitting is the goal. We believe that the TEN (HL) test may play a role in predicting the prognosis of inner ear diseases such as sudden hearing loss, in which high frequency hearing loss is regarded as one of prognostic factors. Additional studies should be performed in the future to verify this hypothesis.

This preliminary study has the following limitations. We did not examine the test-retest reliability to confirm the clinical stability of TEN (HL). Pepler, et al. [13] reported that the repeatability of a DR was up to $97 \%$ among 70 ears; however, they performed the TEN (HL) test in a small sample only. Therefore, additional evaluation of the TEN test in a larger sample is necessary to confirm the stability of the TEN (HL) test. Second, we found that the presence of a DR may be different according to disease etiology. However, this may be hasty conclusions based on preliminary evidences. The diversity of etiologies and unequal sample sizes may act as a confounder in the interpretation of the results of the TEN test. Further studies focusing on the application of the TEN (HL) test according to each disease will help to understand the role of a DR in those diseases. As described above, we assume that the TEN (HL) test may contribute to an expectation of recovery in fluctuating hearing loss such as sudden hearing loss or chronic otitis media after treatment in addition to conventional hearing aid fitting. Third, we did not conduct contralateral masking in patients with asymmetric hearing loss, which might influence the presence of a DR. However, to the best of our knowledge, a standard method for conducting contralateral masking while performing the TEN test has not yet been established. Warnaar and Dreschler [14] conducted contralateral masking with an independently created noise, which was spectrally shaped to the ipsilateral noise at the level of the probe minus $30 \mathrm{~dB}$. However, most studies do not describe the method used to perform contralateral masking to resolve cross-listening. An additional study on the effect of contralateral masking on the results of the TEN test may help to understand the clinical significance of the TEN test.

\section{Conclusion}

Although performance of the TEN test is limited by frequencies and hearing levels, it provides additional information regarding DRs and may therefore be potentially used as a prognostic tool for diverse diseases causing hearing loss.

\section{Acknowledgments}

This research was supported by EMBRI Grants 2013 EMBRIDJ0005 from Eulji University.

\section{REFERENCES}

1) Moore BC, Huss M, Vickers DA, Glasberg BR, Alcántara JI. A test for the diagnosis of dead regions in the cochlea. Br J Audiol 2000;34: 205-24.

2) Moore BC, Glasberg BR, Stone MA. New version of the TEN test with calibrations in dB HL. Ear Hear 2004;25:478-87.

3) Moore BC. Testing for cochlear dead regions: audiometer implementation of the TEN (HL) test. Hear Rev 2010;17:10-6, 48.

4) Cox RM, Alexander GC, Johnson J, Rivera I. Cochlear dead regions in typical hearing aid candidates: prevalence and implications for use of high-frequency speech cues. Ear Hear 2011;32:339-48.

5) Pepler A, Munro KJ, Lewis K, Kluk K. Prevalence of cochlear dead regions in new referrals and existing adult hearing aid users. Ear Hear 2014;35:e99-109.

6) Vinay, Moore BC. Prevalence of dead regions in subjects with sensorineural hearing loss. Ear Hear 2007;28:231-41.

7) Sheehy JL. Vasodilator therapy in sensory-neural hearing loss. Laryngoscope 1960;70:885-914.

8) Kanda Y. Investigation of the freely available easy-to-use software 'EZR' for medical statistics. Bone Marrow Transplant 2013;48:452-8.

9) Papp Z, Rezes S, Jókay I, Sziklai I. Sensorineural hearing loss in chronic otitis media. Otol Neurotol 2003;24:141-4.

10) Summers V, Molis MR, Müsch H, Walden BE, Surr RK, Cord MT. 
Identifying dead regions in the cochlea: psychophysical tuning curves and tone detection in threshold-equalizing noise. Ear Hear 2003;24: $133-42$.

11) Preminger JE, Carpenter R, Ziegler CH. A clinical perspective on cochlear dead regions: intelligibility of speech and subjective hearing aid benefit. J Am Acad Audiol 2005;16:600-13; quiz 631-2.

12) Hornsby BW, Dundas JA. Factors affecting outcomes on the TEN (SPL) test in adults with hearing loss. J Am Acad Audiol 2009;20:
251-63.

13) Pepler A, Munro KJ, Lewis K, Kluk K. Repeatability, agreement, and feasibility of using the threshold equalizing noise test and fast psychophysical tuning curves in a clinical setting. Int J Audiol 2014;53:745-52.

14) Warnaar B, Dreschler WA. Agreement between psychophysical tuning curves and the threshold equalizing noise test in dead region identification. Int J Audiol 2012;51:456-64. 